\title{
DOSSIER \\ The Arthurs Report on Law and Learning / \\ Le Rapport Arthurs sur Le droit et le savoir \\ 1983-2003
}

\section{Editorial}

Twenty years ago the Consultative Group on Research and Education in Law, mandated by Canada's Social Science and Humanities Research Council, published its report Law and Learning / Le droit et le savoir. The document is commonly referred to as the Arthurs Report, after the Consultative Group's Chair, Harry Arthurs. The report's findings on legal scholarship, research and education in Canada and its recommendations received widely varying responses across the country. On the one hand, there were those who accepted the report as a bold and necessary call for change, while at the other extreme were those who resented the potential disruption to the well established comfort, privilege and insularity of the academic and professional legal community. The Canadian Journal of Law and Society owes its creation to the door opened by this watershed event; once solidly established by a multidisciplinary team, joined CLSA/ACDS. Like the editors who preceded me (Rainer Knopff, Claude Thomasset, Roderick A. Macdoald, René Côté and Marie-Andrée Bertrand), I remember these origins, and the varied reactions to the report, some still prevalent today.

When the Canadian Law and Society Association decided to dedicate the 2003 mid-winter meeting to revisiting the Arthurs Report, we wanted to extend the debate to our readership. We invited the participants to submit their papers to $C J L S / R C D S$, and most of them did just that. The result is this dossier. In the first two contributions, Roderick A. Macdonald and Constance Backhouse outline the most important features and recommendations of the Report. They draw particular attention to the unabashed disciplinary insularity of legal research and publication of the time - confined to mostly doctrinal analysis or research for immediate professional use, as well as to the ossified structure and content of the law school curricula and the limited scope of graduate studies in law then in existence. They search out traces of the Report, - some still perceptible, some now lost, discuss the variable impact of the recommendations on different issues and project into the future. Although their assessment shows certain positive developments, the global picture emerging twenty years later seems not very different. Andrée Lajoie, invited to "comment the

Canadian Journal of Law and Society / Revue Canadienne Droit et Société, 2003, Volume 18, no. 1, pp. 1-3 


\section{Ruth Murbach}

comments" of Macdonald and Backhouse, primarily compares their findings to the research and graduate education in francophone civil law faculties, which have been somewhat neglected in both reviews, as they indeed were in the Arthurs Report. The remaining papers go further into specific issues. Diana Majury takes up the law teaching issue, a theme that stirs up less and less interest among law professors and whose structure and content have changed very little. The Arthurs Report had also strongly encouraged legal education outside law faculties. Lesley A. Jacobs justifies its importance and necessary difference by referring to the key concept of legal consciousness: the understanding and experience of legality distinguish legal professionals from ordinary citizens, insiders from outsiders. Our dossier closes with the very current question - at least outside Quebec - of increased law school tuition fees. Jo-Anne Pickel looks at the consequences on access to legal education for students whose interests in law are multidisciplinary and scholarly rather than oriented towards the traditional law firm career.

By publishing this special dossier we not only want to make a tribute to the $20^{\text {th }}$ anniversary of a document that has been widely discussed in the 1980 s and to the eminent author whose signature it carries. We also wish to initiate a wider debate and invite our readers to let us know their reactions and comments, on the questions raised here but also on other dimensions we have not been able to treat adequately. They will be taken up in an upcoming CJLS issue.

\section{Éditorial}

Il y a vingt ans, en 1983, le Groupe consultatif sur la recherche et les études en droit publiait son rapport adressé au Conseil de recherche en sciences humaines du Canada, intitulé Le droit et le savoir / Law and Learning. Il est communément désigné de Rapport Arthurs, référant ainsi à Harry Arthurs qui présidait le Groupe consultatif. La parution de ce rapport sur l'état de la recherche et de l'enseignement du droit au Canada fut alors reçue tantôt comme un bilan sans complaisance invitant au changement, tantôt comme une onde de choc, bouleversant le confort et la distinction de la position particulière, sinon de l'isolement, que la communauté juridique universitaire s'était traditionnellement forgée parmi l'ensemble des disciplines. La Revue Canadienne Droit et Société / Canadian Journal of Law and Society doit sa création par une équipe multidisciplinaire à l'avènement de cet événement et fut associée par la suite à l'Association du même nom. Comme les rédacteurs qui m'ont précédée (Rainer Knopff, Claude Thomasset, Roderick A. Macdonald et Marie-Andrée Bertrand en collaboration avec René Côté), je n'ai pas oublié ce fait, pas plus que les réactions suscitées dont certaines perdurent.

C'est dire que lorsque l'Association canadienne droit et société prit l'initiative de consacrer son colloque d'hiver, en janvier 2003, à la célébration et à la réflexion sur cet anniversaire mémorable, nous voulions l'élargir à l'ensemble de nos lecteurs pour susciter un débat. Nous avons 
invité les participants à soumettre des textes plus élaborés de leurs présentations à la $R C D S / C J L S$. La majorité acceptèrent et ce dossier en est le résultat. Dans les deux premières contributions, Roderick A. Macdonald et Constance Backhouse dressent un bilan qualitatif et quantitatif des grands axes et recommandations du Rapport Arthurs. Ils insistent notamment sur le splendide repli disciplinaire d'une recherche trop confinée à l'exégèse doctrinale sinon à une utilité professionnelle à courte vue, sur la structure et le contenu bétonnés de l'enseignement au premier cycle et la pauvreté des programmes d'études supérieures. Ils suivent les traces perceptibles ou perdues depuis, s'interrogent sur cet impact très variable des recommandations selon les enjeux et se projettent dans l'avenir. S'ils constatent certains acquis, le portrait général ne semble avoir subi que des retouches depuis vingt ans. Andrée Lajoie a été invitée à 'commenter leurs commentaires', ce qu'elle fait dans la perspective de la recherche et de l'enseignement dans les facultés de droit civil, francophones, quelque peu négligées dans ce bilan, tout comme dans celui du Rapport Arthurs, d'ailleurs. Les autres textes du dossier approfondissent des questions particulières soulevées dans le Rapport. Diana Majury reprend le thème de l'enseignement du droit, qui retiendrait de moins en moins l'intérêt des professeurs et dont la structure et le contenu n'ont que très peu changé. Le Rapport Arthurs avait aussi encouragé l'enseignement du droit en dehors des facultés de droit. Lesley A Jacobs justifie son importance à partir du concept clé de la sensibilité juridique, de la compréhension et expérience de la légalité qui distinguent juristes et non juristes. Le dossier se clôt avec la question d'actualité de l'augmentation massive des frais de scolarité dans les facultés de droit du Canada anglais. Jo-Anne Pickel pose la question de ses conséquences sur l'accessibilité à la formation en droit d'étudiants qui ne se dirigent pas nécessairement vers une carrière traditionnelle d'avocat.

La publication de ce dossier vise non seulement à rendre hommage à ce document largement débattu lors de sa parution, il y a vingt ans, et à l'éminent chercheur dont il porte la signature. Bien des dimensions n'ont pu qu'être effleurées ici. Nous souhaitons aussi ouvrir un débat en invitant les lecteurs de la $R C D S$ à nous faire part de leurs réactions et commentaires et nous y reviendrons alors dans une prochaine édition de la revue.

Ruth Murbach

Editor/Rédactrice 